\title{
Article court
}

\section{Immunochimie : quelle place en 2008 ?}

\section{Immunochemistry in 2008}

\author{
Jean-Pierre Goullé ${ }^{1,2 \star}$, Élodie Saussereau ${ }^{1}$, Michel Guerbet ${ }^{2}$, Christian Lacroix $^{1}$ \\ ${ }^{1}$ Laboratoire de pharmacocinétique et de toxicologie Cliniques, Groupe Hospitalier du Havre, BP 24, 76083 Le Havre Cedex, France \\ 2 Laboratoire de Toxicologie, Faculté de Médecine et de Pharmacie, 22 boulevard Gambetta, 76183 Rouen Cedex, France
}

\begin{abstract}
Résumé - Au cours des deux dernières décennies, des avancées déterminantes ont été réalisées dans le domaine de l'analyse toxicologique. Le détecteur de masse s'impose désormais comme l'outil universel, couplé à un chromatographe en phase gazeuse, à un chromatographe en phase liquide ou à un spectromètre à plasma induit. Dans un tel environnement, on pourrait penser que l'immunochimie n'a plus sa place. Il n'en est rien, l'immunoanalyse s'avère être un moyen de dépistage utile dans les différents domaines de la toxicologie, clinique, judiciaire (à l'exception de la recherche de soumission chimique), professionnelle, environnementale. Il s'agit d'une méthode rapide, simple et qui permet le traitement de grandes séries d'échantillons pour un coût réduit. Qu'elle soit utilisée comme moyen de dépistage sur le terrain ou comme méthode de criblage en laboratoire, deux pré-requis s'imposent : l'absence de faux négatif et la confirmation de tout résultat positif par une méthode chromatographique séparative couplée à la spectrométrie de masse.
\end{abstract}

Mots clés : Immunochimie, toxicologie clinique, toxicologie judiciaire

\begin{abstract}
Over the past two decades great progress has been made in analytical toxicology. The development of mass spectrometry as a new technology for gas chromatography, liquid chromatography or induced coupled plasma spectrometry is a major advancement in the field of toxicology. Despite this major progress, immunochemistry still remains up to date. Immunoassays are useful screening techniques and have been successfully applied to various fields of toxicology, i.e. clinical toxicology, forensic toxicology (except for drug facilitated crimes), workplace testing toxicology, and environmental toxicology. Immunoanalysis is quite easy to perform, economic and a highly effective method, especially when numerous samples have to be tested. Used as on-site testing or as a laboratory screening technique, it is mandatory to obtain no false negative results and each positive result must be confirmed by a coupled chromatographymass spectrometry technique.
\end{abstract}

Key words: Immunochemistry, clinical toxicology, forensic toxicology

Reçu le 17 novembre 2008, accepté après modifications le 9 mars 2009

Publication en ligne le 19 avril 2009

\section{Introduction}

Au cours des deux dernières décennies, des avancées déterminantes ont été réalisées dans le domaine de l'analyse toxicologique. Les méthodes chromatographiques ont remplacé les réactions colorimétriques peu spécifiques et peu sensibles. Le détecteur de masse s'impose désormais comme l'outil universel du toxicologue analyste, couplé à un chromatographe en phase gazeuse (GC-MS ou GC-MS/MS), à un chromatographe

\footnotetext{
^ Correspondance : Jean-Pierre Goullé, Tél. 02327332 23,

Fax. 02327332 38,jgoulle@ch-havre.fr
}

en phase liquide (LC-MS/MS), ou à un spectromètre à plasma induit (ICP-MS). Dans ce contexte de haute technologie, à la recherche de performances analytiques améliorées, on pourrait penser que l'immunochimie n'a plus sa place. Il n'en est rien, bien au contraire. Les techniques immunochimiques s'avèrent très utiles dans les différents domaines de l'analyse toxicologique : toxicologie clinique, toxicologie judiciaire, toxicologie professionnelle, toxicologie environnementale. En effet, le délai de mise en œuvre des méthodes chromatographiques n'est pas toujours compatible avec la prise en charge optimale d'une urgence dans une intoxication médicamenteuse par exemple. 
L'analyse d'un grand nombre d'échantillons, dans le cadre du dépistage des stupéfiants au bord de la route ou en santé au travail nécessite la mise en œuvre de tests rapides, faciles à utiliser sur le terrain. Dans ces deux derniers cas, l'immunoanalyse s'avère être un complément indispensable et non un concurrent de l'analyse chromatographique, permettant de limiter le nombre d'examens de confirmation. Dans le domaine judiciaire, si l'intérêt de l'immunochimie n'est plus à démontrer, son usage impose toujours la confirmation par une méthode séparative et spécifique. Ceci explique qu'en 2008, les techniques immunologiques occupent une place de choix dans le secteur de l'industrie du diagnostic. Les possibilités de développement sont importantes dans un marché en pleine expansion et face aux nombreux besoins qu'il s'agisse de tests rapides de terrain ou d'analyses en laboratoire.

\section{Place de l'immunoanalyse sur le marché mondial et national de l'industrie du diagnostic}

Le marché mondial de l'industrie du diagnostic in vitro s'est élevé à 27 milliards d'euros en 2007 [1]. Le premier marché, celui des États-Unis d'Amérique, représente 10,2 milliards d'euros soit $38 \%$, le second est celui de l'Europe (27 pays) estimé à 9,8 milliards d'euros soit $36 \%$, le reste du monde et le Japon avec 7,0 milliards d'euros atteint $26 \%$. Au sein de l'Europe, l'Allemagne avec $21 \%$, la France et l'Italie avec chacune $16 \%$ constituent les trois marchés les plus importants. Le syndicat de l'industrie du diagnostic in vitro qui regroupe 200 entreprises, évalue le marché national en 2007 à 1,59 milliards d'euros [2]. Ce montant est réparti en deux catégories : les ventes aux laboratoires d'analyses pour 1,25 milliards d'euros et les ventes d'autotests pour 0,34 milliards d'euros, composées à $95 \%$ de systèmes d'autosurveillance de la glycémie. Parmi les ventes aux laboratoires, les réactifs, hors autotests, représentent la part la plus importante avec 1,04 milliards, les instruments n'atteignant que 0,21 milliards. En ce qui concerne la répartition des réactifs, en 2007, l'immunochimie arrive très largement en tête avec $34,5 \%$ du total (359 millions d'euros), puis l'immunologie infectieuse avec 22,6\%, l'hématologie avec $19,6 \%$, la biochimie avec $11,9 \%$ et la microbiologie avec $10,3 \%$; les tests génétiques ne représentant qu'environ $1 \%$. Tous ces dispositifs de diagnostic in vitro sont soumis à une réglementation européenne, la directive 98/79 $\mathrm{CE}$ ayant introduit une obligation marquage CE pour tous les dispositifs. Cette directive a été transposée en droit français par une ordonnance de mars 2001 complétée par un décret du 4 février 2004, puis par six arrêtés de novembre 2004 et un arrêté de février 2005. La mise sur le marché des produits est effectuée sous la responsabilité des fabricants qui, en apposant le marquage $\mathrm{CE}$, s'engagent au respect des exigences essentielles de la directive garantissant la qualité des produits et la sécurité des utilisateurs. L'Agence française de sécurité sanitaire des produits de santé (Afssaps) a sous sa responsabilité le contrôle et la surveillance du marché des dispositifs médicaux de diagnostic in vitro. Un système de réactovigilance a été mis en place par l'Afssaps qui exige la déclaration de tout incident ou risque d'incident dû à un dispositif de diagnostic in vitro.
L'agence réalise également des opérations d'évaluation et de contrôle de ces produits pour s'assurer de leur conformité aux exigences de la directive. Les travaux de l'Afssaps sont financés par une taxe de 0,24\% sur le chiffre d'affaires des fabricants commercialisant des produits sur le marché français.

\section{Place de l'immunoanalyse dans les différents domaines de la toxicologie}

L'intérêt de l'immunoanalyse peut également se mesurer au travers des nombreux articles publiés dans les Annales de Toxicologie Analytique dans ce domaine depuis 2000 : qu'il s'agisse de l'évaluation du dépistage de drogues sur le terrain [3], de la place d'un test immunochimique dans le criblage des cannabinoïdes dans le sang total [4], des limites de l'immunoanalyse dans le dépistage urinaire [5], des biomarqueurs de toxicité dans les principales intoxications graves [6], du dépistage de quatre classes de stupéfiants dans les cheveux [7], de l'intérêt d'un immunoessai pour le dépistage urinaire de drogues illicites et de médicaments [8], ou de l'évaluation du dépistage de quatre classes de stupéfiants et des benzodiazépines dans le sang total laqué [9]. Il ne s'agit là que des publications principalement axées sur ce sujet ou pour lesquels l'immunochimie occupe une place très importante.

\subsection{Immunoanalyse et toxicologie clinique en 2008}

Un article de ce numéro étant consacré à la pertinence de l'immunochimie pour les services d'urgence hospitalière, nous ne développerons pas cet aspect. Il est cependant utile de rappeler le consensus clinico-biologique selon lequel l'analyse biologique prime sur l'analyse toxicologique $[6,10]$. En effet, le délai d'obtention des résultats par les méthodes chromatographiques est souvent incompatible avec les contraintes liées à la prise en charge médicale du malade, alors que le bilan biologique de base est disponible rapidement grâce aux automates dont sont équipés tous les laboratoires. Il existe cependant une exception à cette règle, ce sont les dosages de médicaments qui peuvent être réalisés par immunoanalyse, sur l'automate du bilan biologique ou sur un équipement dédié, dans le délai du bilan biologique et disponibles avec ce dernier. Leur quantification est parfois utile, comme pour les anticonvulsivants, voire indispensable pour quelques molécules : digitaliques, méthotrexate, paracétamol, salicylés, théophylline [6]. La recherche qualitative ou semi-quantitative peut s'avérer utile ou précieuse pour deux familles de médicaments, benzodiazépines et antidépresseurs tricycliques, ainsi que pour les principales drogues avec les limites qu'il convient de rappeler quant à l'interprétation des résultats, de la compétence exclusive du biologiste [6]. Mura en dresse la liste dans ce numéro [11]. Dans un contexte, où la prise en charge clinique est prioritaire, l'identification formelle du toxique par une méthode chromatographique, pour confirmer le résultat du dosage effectué par immunoanalyse, n'est pas nécessaire. Cette règle peut également être appliquée à l'interprétation des résultats lors de la recherche d'une famille médicamenteuse. Ainsi, 
dans notre expérience clinico-biologique, le dosage plasmatique semi-quantitatif des antidépresseurs tricycliques à l'admission est un élément indispensable à la prise en charge et à l'orientation du malade [12]. La présence de l'un des trois critères suivants constitue une condition nécessaire mais suffisante pour surveiller le patient dans une structure spécialisée (soins intensifs ou réanimation) : défaillance vitale, signes électriques caractéristiques à l'ECG, concentration en tricycliques exprimée en imipramine supérieure à $500 \mathrm{ng} / \mathrm{mL}$ [12]

D'une manière générale, les immunoessais peuvent être utilisés avec succès en toxicologie clinique tant pour le diagnostic que le dépistage d'une intoxication, ainsi que pour le suivi thérapeutique et toxicologique. C'est ce que montrent Hoizey et Labat avec la cotinine [13], Kintz avec les marqueurs d'éthylisme chronique [14] et Alvarez avec les médicaments substitutifs de l'héroïne et autres opioïdes [15]. Cependant, les limites de ces examens doivent être connues des utilisateurs. Parmi les causes d'erreur il convient de rappeler les interférences possibles liées au milieu biologique (hémolyse, trouble, ictère... ). Les interférences dues à des structures chimiques (autres que celles à rechercher) reconnues par l'anticorps susceptibles de donner une réaction faussement positive ou de majorer le signal doivent être connues (les phénothiazines ou la carbamazépine peuvent majorer la réponse lors de la recherche des antidépresseurs tricycliques par exemple). Les adultérants des tests urinaires constituent également une cause d'erreur [16]. Le niveau du seuil de positivité doit également être choisi de telle sorte que le test ne comporte pas de faux négatif. Ce choix a pour corollaire un plus grand nombre de faux positifs. Dans tous les cas, seules les méthodes chromatographiques séparatives (GC-MS, LC-MS/MS), permettent de confirmer la présence d'un xénobiotique, médicament ou drogue.

\subsection{Immunoanalyse et toxicologie judiciaire en 2008}

Si l'immunoanalyse peut également s'avérer utile en toxicologie judiciaire, son emploi doit impérativement obéir à des règles différentes de celles qui prévalent en toxicologie clinique. En effet, tout résultat positif ou négatif, obtenu par un immunoessai doit obligatoirement être validé par une méthode de confirmation sous peine de nullité du rapport d'expertise toxicologique. L'immunoanalyse ne peut donc être utilisée en médecine légale que comme une méthode de dépistage et d'orientation uniquement à l'usage de l'expert analyste. Son emploi doit donc être proscrit au pied de la table d'autopsie, ainsi que dans tout laboratoire ne disposant pas de l'équipement requis pour réaliser les confirmations. C'est dans ce domaine de la toxicologie que l'usage de l'immunoanalyse s'avère la plus délicate. Outre le sang et les urines, milieux traditionnels analysés en biologie médicale, la salive et les phanères présentent un intérêt majeur dans un cadre médico-légal. Cependant, l'emploi des immunoessais pose de nombreux problèmes spécifiques à ces matrices. Dans ces milieux, contrairement aux urines, en raison de propriétés pharmacocinétiques particulières, ce sont le plus souvent les molécules mères qui caractérisent le profil d'exposition à une substance. Or, les tests d'immunoanalyse ont été développés pour reconnaître les métabolites urinaires et non les principes actifs. Par ailleurs, le sang prélevé en biologie médicale donne après centrifugation du sérum ou du plasma limpide, ce qui est primordial pour une immunoanalyse de qualité. Ceci est très rarement le cas pour le sang prélevé sur un cadavre qui ne permet d'obtenir que du sérum plus ou moins hémolysé, voire uniquement du sang laqué, incompatible avec l'emploi de nombreux immunoessais. D'autre part les tests utilisés dans un contexte judiciaire sont destinés à des usages variés : test rapides à lecture visuelle directe d'exécution facile sur le terrain, immunoessais pour laboratoire nécessitant un équipement de lecture. Verstraete et Labat font le point dans ce numéro sur l'utilisation de tests rapides salivaires au bord de la route et en santé au travail [17]. Cirimèle propose d'appliquer des méthodes immunologiques pour le dépistage des xénobiotiques dans les cheveux [18], dans le prolongement du dépistage de quatre classes de stupéfiants par une technique immuno-enzymatique par la même équipe [7]. Pépin a décrit quant à lui le criblage des stupéfiants dans le sang total par immunochimie [19]. Dès 2001, Kintz et coll. [4] montraient l'intérêt d'un tel test immunochimique de dépistage des cannabinoïdes dans le sang total.

En ce qui concerne l'emploi de l'immunoanalyse en toxicologie judiciaire, il convient de considérer deux cas de figure et les pré-requis correspondants :

- le dépistage d'une conduite après avoir consommé un stupéfiant, dans le cadre de la sécurité routière : les tests utilisés, qu'ils soient urinaires ou salivaires, doivent être exempts de faux négatifs. Dans le cas contraire, ceci entrâ̂nerait l'arrêt de la procédure, alors que le conducteur pouvait avoir consommé un stupéfiant. Si le dépistage utilisé ne comporte pas de faux négatif, tous les sujets positifs, donc susceptibles d'avoir consommé subiront une prise de sang, dont seule l'analyse a force de loi ;

- le criblage des stupéfiants dans le sang total par immunochimie nécessite quant à lui deux pré-requis : l'absence de faux négatif, ce qui conduirait à arrêter la procédure alors que le sujet avait consommé un stupéfiant, mais également la confirmation de tous les résultats positifs par une méthode chromatographique séparative (GC-MS, LC-MS/MS).

L'emploi d'une technique d'immunoanalyse de type ELISA pour le criblage sanguin des stupéfiants, sous réserve de l'absence de faux négatif, ne nécessite pas a priori de confirmation par une méthode séparative. Tout au plus, il peut être exigé de réaliser ces analyses en double comme cela est le cas au Royaume Uni [20]. Outre le fait que les échantillons doivent être analysés deux fois, le début et la fin de chaque série comportent obligatoirement au minimum trois calibrateurs (négatif, positif, seuil de positivité). Il est d'ailleurs recommandé d'utiliser une courbe de calibration.

Dans notre expérience, après avoir réalisé à la fois le criblage sanguin par immunoanalyse, puis la quantification par GC-MS sur plusieurs milliers d'échantillons, nous avons constaté pour les cannabinoïdes, contrairement aux trois autres familles, de rares faux négatifs. Il s'agissait toujours d'échantillons montrant de faibles concentrations de THC-COOH avec un THC voisin de 0,5 à $1,0 \mathrm{ng} / \mathrm{mL}$. Ceci rejoint la constatation faite dès 2001 par Kintz et coll. [4]. 
Tableau I. Durée de détectabilité dans les urines par différentes techniques après ingestion d'une unité galénique [21].

\begin{tabular}{ccccccc}
\hline Molécule & Immuno sauf CEDIA & CEDIA & LC-DAD & GC-MS & LC-MS & LC-MS/MS \\
\hline lorazépam & Non détecté & $96 \mathrm{~h}$ & Non détecté & $84 \mathrm{~h}$ & $84 \mathrm{~h}$ & $>144 \mathrm{~h}$ \\
OH-bromazépam & Non détecté & $/$ & $36 \mathrm{~h}$ & $24 \mathrm{~h}$ & $108 \mathrm{~h}$ & $144 \mathrm{~h}$ \\
bromazépam & Non détecté & $48 \mathrm{~h}$ & $12 \mathrm{~h}$ & $72 \mathrm{~h}$ & $48 \mathrm{~h}$ & $>144 \mathrm{~h}$ \\
zopiclone & Non détecté & Non détecté & $12 \mathrm{~h}$ & dégradée & $36-48 \mathrm{~h}$ & $120 \mathrm{~h}$ \\
7-NH -flunitrazépam & Non détecté & $48 \mathrm{~h}$ & $36 \mathrm{~h}$ & $48 \mathrm{~h}$ & $96 \mathrm{~h}$ & $144 \mathrm{~h}$ \\
zolpidem & Non détecté & Non détecté & $24 \mathrm{~h}$ & $12 \mathrm{~h}$ & $36-60 \mathrm{~h}$ & $48-96 \mathrm{~h}$ \\
7-NH-2-clonazépam & Non détecté & $96 \mathrm{~h}$ & $12 \mathrm{~h}$ & $132 \mathrm{~h}$ & $144 \mathrm{~h}$ & $144 \mathrm{~h}$ \\
\hline
\end{tabular}

Dans le prolongement des pratiques en toxicologie clinique, où des coffrets de réactifs destinés aux urines sont utilisés avec du sérum, il peut être tentant de les employer avec du sang. Avec les techniques non ELISA qui ne comportent pas d'étape de lavage permettant d'éliminer la matrice biologique susceptible d'interférer, l'analyse sur le sang doit impérativement être proscrite. Dans ce cas, pour limiter ces effets de matrice, un prétraitement de l'échantillon faisant appel à une précipitation, le plus souvent par l'acétone ou l'acétonitrile, est parfois proposé. Ces techniques ne sont pas conseillées et l'utilisation en médecine légale est proscrite par les fabricants.

Cas particulier de la soumission chimique : la soumission chimique fournit un très bel exemple des limites de l'immunoanalyse dans la détection urinaire de faibles concentrations de benzodiazépines et de dérivés apparentés. L'étude expérimentale in vivo, sur 12 volontaires sains ayant consommé chacun une unité galénique des principes actifs suivants : lorazépam 2,5 mg, bromazépam $6 \mathrm{mg}$, flunitrazépam $1 \mathrm{mg}$, zolpidem $10 \mathrm{mg}$, clonazépam $2 \mathrm{mg}$, zopiclone $7,5 \mathrm{mg}$, montre qu'aucune technique immunochimique, ne peut être utilisée pour le dépistage de la soumission chimique comme le rappelle le tableau I [21].

\subsection{Immunoanalyse et toxicologie professionnelle et environnementale en 2008}

La plupart des constats et remarques faits dans les paragraphes précédents sont applicables en toxicologie professionnelle et environnementale. À l'heure actuelle si l'utilisation de tests rapides en surveillance professionnelle progresse lentement, nous manquons de tests immunochimiques dans le cadre de la surveillance environnementale, vaste domaine, ouvert à de nombreux développements. Hoizey et Labat [13] ainsi que Mouthon [22] consacrent un article à des applications sur ces thèmes.

\section{Conclusion}

Face au développement des méthodes séparatives, l'immunoanalyse s'avère être aujourd'hui un complément indispensable dans les domaines du dépistage, du diagnostic et du traitement. Les besoins et les attentes des analystes, mais également d'autres catégories de professionnels sont nombreux, ouvrant aux industriels un marché à fort potentiel. Il convient cependant d'être vigilant sur la diversité des réponses des différentes molécules d'une même classe vis-à-vis d'un anticorps, qui doit inciter à la prudence dans l'interprétation d'un test immunologique, mais également dans le choix du seuil de positivité. Que l'immunochimie soit utilisée comme moyen de dépistage rapide sur le terrain ou comme méthode de criblage au laboratoire, deux pré-requis s'imposent : l'absence de faux négatif et la confirmation de tout résultat positif par une méthode chromatographique séparative couplée à la spectrométrie de masse. Si ces conditions sont remplies, l'immunoanalyse s'avère être une méthode de dépistage rapide, simple permettant le traitement de grandes séries d'échantillons tout en bénéficiant d'un coût réduit.

\section{Références}

1. Chiffres clés de l'industrie du diagnostic in vitro 2008. Syndicat de l'industrie du diagnostic in vitro. Edition novembre 2008.

2. Site internet www.sfrl.fr consulté le 27/10/2008.

3. Samyn N, Areschka V, Verstraete A. Évaluation de tests de dépistage des drogues sur le terrain. Ann Toxicol Anal. 2000; 12(2): 105-115.

4. Kintz P, Lohner S, Sengler C, Cirimele V. Place d'un test immunochimique dans le criblage des cannabinoïdes dans le sang total. Ann Toxicol Anal. 2001; 13(4): 284-285.

5. Capolaghi B. Dépistage urinaire : les limites de l'immunoanalyse. Ann Toxicol Anal. 2002; 14(1): 24-26.

6. Goullé JP et coll. Biomarqueurs de toxicité et anomalies métaboliques dans les principales intoxications graves. Ann Toxicol Anal. 2003; 15(3): 208-220.

7. Pujol ML, Tritsch PJ, Cirimele V, Kintz P. Dépistage de quatre classes de stupéfiants dans les cheveux par technique ELISA à l'aide du test One-Step ${ }^{\mathrm{TM}}$ et confirmation par chromatographie gazeuse couplée à la spectrométrie de masse. Ann Toxicol Anal. 2006; 18(4): 291-296.

8. Mura P, Papet Y, Brunet B. Intérêt du Triage ${ }^{\circledR}$ TOX Drug Screen pour le dépistage urinaire de drogues illicites et de médicaments. Ann Toxicol Anal. 2007; 19(3): 221-225.

9. Grassin Delyle S, Mathieu B, Abe E, Alvarez JC. Évaluation du dépistage de quatre classes de stupéfiants et des benzodiazépines dans le sang total laqué par l'analyseur Evidence Investigator ${ }^{\circledR}$. Ann Toxicol Anal. 2008; 20(1): 17-24.

10. Capolaghi B, Moulsma M, Houdret N, Baud FJ. Stratégies analytiques en toxicologie d'urgence. Ann Toxicol Anal. 2000; 12(4): 274-281. 
11. Brunet B et coll. Pertinence de l'immunochimie pour les services d'urgence hospitalière. Ann Toxicol Anal. 2009; 21(1): 37-43.

12. Goulé JP, Rigaud JP, Lacroix C, Nouveau J. Intoxications graves par antidépresseurs tricycliques. À propos de 38 observations. Toxicorama. 1998; 10(4): 255-267.

13. Hoizey G, Labat L. Cotinine en pédiatrie et en santé au travail. Ann Toxicol Anal. 2009; 21(1): 27-35.

14. Kintz P et coll. Les marqueurs de l'éthylisme chronique. Focus sur les approches immuno-chimiques. Ann Toxicol Anal. 2009; 21(1): 21-25.

15. Alvarez JC. Le dépistage immunochimique des médicaments substitutifs de l'héroïne et autres opioïdes. Ann Toxicol Anal. 2009; 21(1): 13-19.

16. Dumestre-Toulet V, Verstraete AG. Les adultérants des tests urinaires. Toxicorama. 1999; 11(3): 155-166.
17. Verstraete A, Labat L. Utilisation des tests rapides de détection de drogues dans la salive au bord de la route et en santé au travail. Ann Toxicol Anal. 2009; 21(1): 3-8.

18. Cirimele $\mathrm{V}$ et coll. Utilisation en routine des tests ELISA One-Step $^{T M}$ (International Diagnostic Systems Corp.) pour le dépistage des xénobiotiques dans les cheveux : bilan de 2 ans d'activité. Ann Toxicol Anal. 2009; 21(1): 9-12.

19. Pépin G. Journée immunochimie de la Société Française de Toxicologie Analytique. Paris, 11 décembre 2008.

20. Basic requirements in toxicology in forensic cases. Forensic standards guidelines. United Kingdom.

21. Kintz P. Soumission chimique : Prise en charge toxicologique. Consensus de la Société Française de Toxicologie Analytique. Ann Toxicol Anal. 2003; 14(4): 239-242.

22. Mouthon G. Immunoanalyse et sécurité des produits d'origine animale. Ann Toxicol Anal. 2009; 21(1): 45-48. 